\title{
INTERPOLATION BY PROPER HOLOMORPHIC EMBEDDINGS OF THE DISC INTO $\mathbb{C}^{2}$
}

\author{
Josip GLobevnik \\ Dedicated to the memory of my mother
}

\section{The result}

Let $\Delta$ be the open unit disc in $\mathbb{C}$. A map $f: \Delta \rightarrow \mathbb{C}^{2}$ is called a proper holomorphic embedding if it is a holomorphic immersion which is one to one and such that the preimage of every compact set is compact. If $f: \Delta \rightarrow \mathbb{C}^{2}$ is a proper holomorphic embedding then $f(\Delta)$ is a closed submanifold of $\mathbb{C}^{2}$ which is, via $f$, biholomorphically equivalent to $\Delta$.

It is not trivial to prove that there are proper holomorphic embeddings from $\Delta$ to $\mathbb{C}^{2}[\mathrm{St}, \mathrm{A}, \mathrm{GS}]$. It is known that given a discrete set $E \subset \mathbb{C}^{2}$ there is a proper holomorphic embedding $f: \Delta \rightarrow \mathbb{C}^{2}$ such that $E \subset f(\Delta)$ [FGS]. In the present paper we prove a stronger result:

Theorem 1.1 Given a discrete set $S \subset \Delta$ and a proper injection $\varphi: S \rightarrow \mathbb{C}^{2}$ there is a proper holomorphic embedding $f: \Delta \rightarrow \mathbb{C}^{2}$ that extends $\varphi$.

In other words, given an injective sequence $\left\{\zeta_{j}\right\} \subset \Delta$ such that $\left|\zeta_{j}\right| \rightarrow 1$ and an injective sequence $\left\{w_{j}\right\} \subset \mathbb{C}^{2}$ such that $\left|w_{j}\right| \rightarrow+\infty$ there is a proper holomorphic embedding $f: \Delta \rightarrow \mathbb{C}^{2}$ such that $f\left(\zeta_{j}\right)=w_{j}(j \in \mathbb{N})$.

The proof of the Carleman approximation theorem of Buzzard and Forstneric $[\mathrm{BFo}]$ can be adapted to prove such a result for proper holomorphic embeddings $f: \mathbb{C} \rightarrow \mathbb{C}^{2}$. In the proof there one uses the fact that $\mathbb{C}$ admits particularly simple embeddings into $\mathbb{C}^{2}$ of the form $\zeta \rightarrow(\zeta, a(\zeta))$ where $a$ is an entire function. There are no such embeddings for $\Delta$ so a different proof is necessary in our case. In the induction step of our proof we use simultaneous composition by automorphisms on the left and on the right, a novelty introduced by Buzzard and Forstnerič.

\section{The scheme of the proof}

Suppose that $S \subset \Delta$ is a discrete set and let $\varphi: S \rightarrow \mathbb{C}^{2}$ be a proper injection. With no loss of generality assume that $S$ is infinite.

Denote by $\mathbb{B}$ the open unit ball in $\mathbb{C}^{2}$. We shall construct inductively a sequence $K_{n}$ of compact subsets of $\Delta$, such that $b K_{n}$ is a smooth Jordan curve

Received January 3, 2002. 
for each $n \in \mathbb{N}$ and such that $K_{n} \subset \subset K_{n+1}(n \in \mathbb{N}), \cup_{n=1}^{\infty} K_{n}=\Delta$, an increasing sequence $r_{n}$ of positive numbers converging to $+\infty$, a decreasing sequence $\varepsilon_{n}$ of positive numbers and a sequence $f_{n}$ of holomorphic maps from $\Delta$ to $\mathbb{C}^{2}$ which are one to one and regular and such that the following hold:

(i) $\varphi\left(\left(\Delta \backslash K_{n}\right) \cap S\right) \subset \mathbb{C}^{2} \backslash r_{n} \overline{\mathbb{B}}$

(ii) $f_{n}\left(\Delta \backslash \operatorname{Int} K_{n}\right) \subset \mathbb{C}^{2} \backslash r_{n} \overline{\mathbb{B}}$

(iii) $f_{n+1}\left(\Delta \backslash K_{n}\right) \subset \mathbb{C}^{2} \backslash r_{n-1} \overline{\mathbb{B}}$

(iv) $f_{n}\left|K_{n} \cap S=\varphi\right| K_{n} \cap S$

(v) $\left|f_{n+1}-f_{n}\right|<\varepsilon_{n} / 2^{n}$ on $K_{n}$

(vi) If $h$ is a holomorphic map on $\operatorname{Int} K_{n}$ that satisfies $\left|h-f_{n}\right|<\varepsilon_{n}$ on $\operatorname{Int} K_{n}$, then $h$ is one to one and regular on $K_{n-1}$

(vii) $(1-1 / n) \Delta \subset K_{n}$

Suppose for a moment that we have done this. By (v) and (vii) $f_{n}$ converges, uniformly on compacta in $\Delta$, to a holomorphic map $f$. By (v), $\left|f_{n}-f\right| \leq$ $\sum_{j=n}^{\infty}\left|f_{j+1}-f_{j}\right| \leq \sum_{j=n}^{\infty} \varepsilon_{j} / 2^{j}<\varepsilon_{n}$ on $K_{n}$ which implies by (vi) that $f$ is regular and one to one on $K_{n-1}$. As this holds for every $n$ it follows that $f$ is regular and one to one on $\Delta$. By (iv), $f$ extends $\varphi$. Let $\zeta \in K_{n+1} \backslash K_{n}$. By (v), $\left|f_{j+1}(\zeta)-f_{j}(\zeta)\right|<\varepsilon_{j} / 2^{j}(j \geq n+1)$ which, by (iii) implies that $|f(\zeta)| \geq$ $\left|f_{n+1}(\zeta)\right|-\sum_{j=n+1}^{\infty}\left|f_{j+1}(\zeta)-f_{j}(\zeta)\right| \geq r_{n-1}-\sum_{j=n+1}^{\infty} \varepsilon_{j} / 2^{j} \geq r_{n-1}-\varepsilon_{n+1}$. This holds for every $n$. Since $r_{n}$ increase to $+\infty$ and since $\varepsilon_{n}$ are decreasing it follows that the map $f$ is proper. Thus, $f$ has all the required properties.

In the process we shall also construct two sequences $S_{n}, T_{n}$ of positive numbers such that $S_{n+1}=S_{n}$ for even $n$ and $T_{n+1}=T_{n}$ for odd $n$. Each map $f_{n}$ will be of the form $f_{n}=A_{n} \circ g_{n}$ where $A_{n}$ is a holomorphic automorphism of $\mathbb{C}^{2}$ and $g_{n}$ is a one to one and regular holomorphic map from an open neighbourhood $U_{n}$ of $\bar{\Delta}$ to $\mathbb{C}^{2}$ which, for even $n$ is transverse to $\left\{(z, w):|z|=S_{n}\right\}$ and satisfies $g_{n}^{-1}\left(\left\{|z|=S_{n}\right\}\right)=b \Delta$, and for odd $n$, is transverse to $\left\{(z, w):|w|=T_{n}\right\}$ and satisfies $g_{n}^{-1}\left(\left\{|w|=T_{n}\right\}\right)=b \Delta$.

With no loss of generality assume that $0 \notin S$. To begin the induction, let $f_{1}(\zeta)=(0, \zeta)$ and let $r_{1}, 0<r_{1}<1 / 2$ be such that $2 r_{1} \bar{\Delta}$ contains no point of $S$. Put $K_{0}=r_{1} \bar{\Delta}, K_{1}=2 r_{1} \bar{\Delta}$. Then (i), (ii) and (vii) are satisfied for $n=1$ and (iv) is vacuously satisfied for $n=1$. Put $S_{1}=T_{1}=1$ and $A_{1}=$ Id so that $f_{1}=A_{1} \circ g_{1}$ where $g_{1}(\zeta)=(0, \zeta)$ and $U_{1}=\mathbb{C}$. Clearly $g_{1}$ is transverse to $\left\{|w|=T_{1}\right\}$ and $g_{1}^{-1}\left(\left\{|w|=T_{1}\right\}\right)=b \Delta$. Put $r_{0}=r_{1} / 2$. Then $A_{1}\left(\left\{|w|>T_{1} / 2\right\}\right)$ misses $2 r_{0} \mathbb{B}$. Put $\varepsilon_{0}=\min \left\{1, r_{1} / 2\right\}$.

Given $f_{n}=A_{n} \circ g_{n}$ we shall have $f_{n+1}=A_{n+1} \circ g_{n+1}$ with $A_{n+1}=\Psi_{n+1} \circ$ $\Theta_{n+1} \circ A_{n}$ where $\Theta_{n+1}$ and $\Psi_{n+1}$ are holomorphic automorphisms of $\mathbb{C}^{2}$ and with $g_{n+1}=G_{n+1} \circ g_{n} \circ p_{n+1}$ where $p_{n+1}$ is a conformal map from a neighbourhood $U_{n+1}$ of $\bar{\Delta}$ to $p_{n+1}\left(U_{n+1}\right) \subset \mathbb{C}$ which is a slight perturbation of the identity on $\bar{\Delta}$ and $G_{n+1}$ is an automorphism of $\mathbb{C}^{2}$ of the form

$$
G_{n+1}(z, w)=\left(z+S_{n+1}\left(\frac{w}{T_{n}}\right)^{M_{n+1}}, w\right) \text { if } n \text { is odd, }
$$




$$
G_{n+1}(z, w)=\left(z, w+T_{n+1}\left(\frac{z}{S_{n}}\right)^{M_{n+1}}\right) \text { if } n \text { is even. }
$$

\section{The induction step, Part 1}

Suppose for a moment that we have constructed $f_{n}=A_{n} \circ g_{n}, K_{n}, S_{n}, T_{n}, r_{n}$ and $\varepsilon_{n-1}$. We want to show how to obtain $\varepsilon_{n}, K_{n+1}, S_{n+1}, T_{n+1}, r_{n+1}$ and $f_{n+1}=A_{n+1} \circ g_{n+1}$. Suppose that $n$ is odd so that $g_{n}: U_{n} \rightarrow \mathbb{C}^{2}$ is transverse to $\left\{(z, w):|w|=T_{n}\right\}$ and satisfies $g_{n}^{-1}\left(\left\{|w|=T_{n}\right\}\right)=b \Delta$. Put $T_{n+1}=T_{n}$. Since $g_{n}$ is transverse to $\left\{|w|=T_{n}\right\}$ and since $S$ is discrete one can, after shrinking $U_{n}$ if necessary, choose $T_{n 1}, T_{n 2}, T_{n 3}$ such that

$$
\frac{T_{n}}{2}<T_{n 3}<T_{n 2}<T_{n 1}<T_{n}
$$

where $T_{n 3}$ is so close to $T_{n}$ that for all $T, T_{n 3} \leq T \leq T_{n}, g_{n}$ is transverse to $\{|w|=T\}$ and $g_{n}^{-1}(\{|w|=T\})$ is a smooth Jordan curve, that

$$
K_{n} \subset g_{n}^{-1}\left\{|w|<T_{n 3}\right\},
$$

that $g_{n}^{-1}\left(\left\{T_{n 3} \leq|w| \leq T_{n 2}\right\}\right)$ contains no point of $S$, and that $g_{n}^{-1}\left(\left\{|w|<T_{n 1}\right\}\right)$ contains a point in $S$ that does not belong to $K_{n}$. Put

$P_{n+1}=g_{n}^{-1}\left(\left\{|w| \leq T_{n 3}\right\}\right), Q_{n+1}=g_{n}^{-1}\left(\left\{|w| \leq T_{n 2}\right\}\right), K_{n+1}=g_{n}^{-1}\left(\left\{|w| \leq T_{n 1}\right\}\right)$.

With no loss of generality assume that $T_{n 3}$ has been chosen so close to $T_{n}$ that (vii) holds with $n$ replaced by $n+1$. We have

$$
K_{n} \subset \subset P_{n+1} \subset \subset Q_{n+1} \subset \subset K_{n+1} .
$$

Clearly $b K_{n+1}$ is a smooth Jordan curve.

By (i), $r_{n}<\min \left\{|\varphi(w)|: w \in\left(\Delta \backslash K_{n}\right) \cap S\right\}$. Thus, one can choose $r_{n+1}>r_{n}$ such that

$\min \left\{|\varphi(w)|: w \in\left(\Delta \backslash K_{n+1}\right) \cap S\right\}-1<r_{n+1}<\min \left\{|\varphi(w)|: w \in\left(\Delta \backslash K_{n+1}\right) \cap S\right\}$.

Then (i) is satisfied with $n$ replaced by $n+1$. Choose $\varepsilon_{n}, 0<\varepsilon_{n}<\varepsilon_{n-1}$, such that

$$
\varepsilon_{n}<r_{n}-r_{n-1}, \quad \varepsilon_{n}<r_{n-1},
$$

and such that (vi) holds. Since $f_{n}$ is one to one and regular on $\Delta$ this is possible by a lemma of Narasimhan [Na, p. 926].

Choose $R, R>2 r_{n+1}, R>2 r_{n}+\varepsilon_{n}$, so large that $f_{n}\left(K_{n}\right)+\mathbb{B} \subset R \mathbb{B}$ and that $\varphi\left(K_{n+1} \cap S\right) \subset R \mathbb{B}$. We need the following lemma.

Lemma 3.1. Let $R>0$ and let $w_{1}, w_{2}, \cdots w_{n} \in R \mathbb{B}, w_{i} \neq w_{j}(i \neq j)$. Given $\gamma>0$ there is a $\delta>0$ such that whenever $q_{1}, q_{2}, \cdots q_{n} \in \mathbb{C}^{2}$ satisfy $\left|q_{i}-w_{i}\right|<\delta, 1 \leq i \leq n$, there is a holomorphic automorphism $\Psi$ of $\mathbb{C}^{2}$ such that:

(i) $\Psi\left(q_{i}\right)=w_{i} \quad(1 \leq i \leq n)$

(ii) $|\Psi(w)-w|<\gamma \quad(w \in R \mathbb{B})$. 
Lemma 3.1 provides a $\theta_{n}, 0<\theta_{n}<\varepsilon_{n} / 2^{n+2}$, such that $\left\{\begin{array}{l}\text { whenever } \psi: K_{n+1} \cap S \rightarrow \mathbb{C}^{2} \text { satisfies }|\psi-\varphi|<3 \theta_{n} \text { on } \\ K_{n+1} \cap S \text { there is a holomorphic automorphism } \Psi \text { of } \mathbb{C}^{2} \text { such that } \\ \Psi \circ \psi=\varphi \mid K_{n+1} \text { and such that }|\Psi-\mathrm{Id}|<\varepsilon_{n} / 2^{n+1} \text { on } R \mathbb{B} .\end{array}\right.$

By (3.2) we may assume that

$$
r_{n}-3 \theta_{n}>r_{n-1}+\varepsilon_{n}+\theta_{n}, \quad 2 r_{n-1}-\theta_{n}>r_{n-1}+\varepsilon_{n}+\theta_{n} .
$$

\section{Proof of Lemma 3.1}

Sublemma 4.1 Suppose that $R>0$ and let $\alpha_{1}, \cdots \alpha_{n} \in R \Delta, \alpha_{i} \neq \alpha_{j}(i \neq j)$. There are $\eta>0$ and $L<\infty$ such that whenever $\beta_{1}, \cdots \beta_{n}$ satisfy $\left|\beta_{i}-\alpha_{i}\right|<$ $\eta, 1 \leq i \leq n$, then for every $j, 1 \leq j \leq n$, there is a polynomial $Q_{j}$ such that (i) $Q_{j}\left(\beta_{i}\right)=\delta_{j i}(1 \leq i, j \leq n)$ (ii) $\left|Q_{j}(\zeta)\right| \leq L(\zeta \in 2 R \Delta)$.

Proof. Choose $\eta>0$ so small that $\alpha_{i}+\eta \Delta \subset R \Delta(1 \leq i \leq n)$ and let $\left|\beta_{i}-\alpha_{i}\right|<$ $\eta(1 \leq i \leq n)$. For each $j, 1 \leq j \leq n$, the polynomial

$$
Q_{j}(\zeta)=\prod_{k=1, k \neq j}^{n} \frac{\zeta-\beta_{k}}{\beta_{j}-\beta_{k}}
$$

satisfies (i). If $|\zeta|<2 R$ then

$$
\left|Q_{j}(\zeta)\right| \leq \frac{(3 R)^{n-1}}{\left(\min _{j \neq k}\left|\beta_{j}-\beta_{k}\right|\right)^{n-1}} .
$$

Now, let $\gamma=\min _{j \neq k}\left|\alpha_{j}-\alpha_{k}\right|$. Passing to a smaller $\eta$ we may assume that $0<\eta<\gamma / 2$. If $\left|\alpha_{i}-\beta_{i}\right|<\eta, 1 \leq i \leq n$, then $\min _{j \neq k}\left|\beta_{j}-\beta_{k}\right| \geq \gamma-2 \eta>0$ so $Q_{j}$ satisfies (ii) with $L=[3 R /(\gamma-2 \eta)]^{n-1}$. This completes the proof.

Proof of Lemma 3.1. Choose a coordinate system in $\mathbb{C}^{2}$ such that if $w_{i}=$ $\left(w_{i}^{1}, w_{i}^{2}\right)$ then $w_{i}^{1} \neq w_{j}^{1}, w_{i}^{2} \neq w_{j}^{2}$ if $i \neq j, 1 \leq i, j \leq n$. By Sublemma 4.1 there are $\eta>0$ and $L<\infty$ such that whenever $\beta_{i}^{1}$ satisfy $\left|\beta_{i}^{1}-w_{i}^{1}\right|<\eta$ and $\beta^{2}$ satisfy $\left|\beta_{i}^{2}-w_{i}^{2}\right|<\eta, 1 \leq i \leq n$, then for each $j, 1 \leq j \leq n$, there are polynomials $Q_{j}^{1}$ and $Q_{j}^{2}$ such that $Q_{j}^{1}\left(\beta_{j}^{1}\right)=1, Q_{j}^{1}\left(\beta_{i}^{1}\right)=0(i \neq j)$, $Q_{j}^{2}\left(\beta_{j}^{2}\right)=1, Q_{j}^{2}\left(\beta_{i}^{2}\right)=0(i \neq j)$ and $\left|Q_{j}^{1}\right|<L,\left|Q_{j}^{2}\right|<L$ on $2 R \Delta$. Let $\left|z_{j}-w_{j}\right|<\eta, 1 \leq j \leq n$. Our map $\Phi$ will be of the form $\Phi=T \circ S$ where $T, S$ are the automorphisms of $\mathbb{C}^{2}$

$$
T(\xi, \zeta)=\left(\xi, \zeta+Q_{1}(\xi)\right), \quad S(\xi, \zeta)=\left(\xi+Q_{2}(\zeta), \zeta\right)
$$

such that

$$
\begin{gathered}
S(R \Delta \times R \Delta) \subset(2 R \Delta) \times(R \Delta), \\
|S(\xi, \zeta)-(\xi, \zeta)|<\gamma / 2 \quad\left((\xi, \zeta) \in(R \Delta)^{2}\right), \\
|T(\xi, \zeta)-(\xi, \zeta)|<\gamma / 2 \quad((\xi, \zeta) \in(2 R \Delta) \times(R \Delta)),
\end{gathered}
$$


and

$$
S\left(z_{i}^{1}, z_{i}^{2}\right)=\left(w_{i}^{1}, z_{i}^{2}\right), \quad T\left(w_{i}^{1}, z_{i}^{2}\right)=\left(w_{i}^{1}, w_{i}^{2}\right) \quad(1 \leq i \leq n) .
$$

By (4.1)-(4.4) the map $\Phi$ satisfies (i) and (ii) in Lemma 3.1. To construct $S$, put $\beta_{j}^{2}=z_{j}^{2}, 1 \leq j \leq n$, and let $Q_{j}^{2}, 1 \leq j \leq n$, be as above. In particular, $Q_{j}^{2}\left(z_{i}^{2}\right)=\delta_{j i}, 1 \leq i, j \leq n$. Put

$$
Q_{2}(\zeta)=\sum_{j=1}^{n}\left(w_{j}^{1}-z_{j}^{1}\right) Q_{j}^{2}(\zeta) .
$$

We have

$$
Q_{2}\left(z_{j}^{2}\right)=\sum_{i=1}^{n}\left(w_{i}^{1}-z_{i}^{1}\right) Q_{i}^{2}\left(z_{j}^{2}\right)=w_{j}^{1}-z_{j}^{1}
$$

and so $S\left(z_{i}^{1}, z_{i}^{2}\right)=\left(z_{i}^{1}+w_{i}^{1}-z_{i}^{1}, z_{i}^{2}\right)=\left(w_{i}^{1}, z_{i}^{2}\right)$. We have

$$
\left|Q_{2}(\zeta)\right| \leq n \cdot \max _{1 \leq j \leq n}\left|w_{j}^{1}-z_{j}^{1}\right| \cdot L, \quad(|\zeta|<R)
$$

which implies that

$$
|S(\xi, \zeta)-(\xi, \zeta)|=\left|\left(Q_{2}(\zeta), 0\right)\right| \leq n \cdot L \cdot \max _{1 \leq j \leq n}\left|w_{j}-z_{j}\right|, \quad(|\zeta|<R) .
$$

In particular, if $\eta>0$ is small enough then $\left|Q_{2}(\zeta)\right|<R,(|\zeta|<R)$, so that (4.1) and (4.2) hold. To construct $T$, put $\beta_{j}^{1}=w_{j}^{1}, 1 \leq j \leq n$, and let $Q_{j}^{1}, 1 \leq j \leq n$, be as above. Put

$$
Q_{1}(\zeta)=\sum_{j=1}^{n}\left(w_{j}^{2}-z_{j}^{2}\right) Q_{j}^{1}(\zeta) .
$$

We have $Q_{1}\left(w_{j}^{1}\right)=w_{j}^{2}-z_{j}^{2} \quad(1 \leq j \leq n)$, so $T\left(w_{i}^{1}, z_{i}^{2}\right)=\left(w_{i}^{1}, z_{i}^{2}+w_{i}^{2}-z_{i}^{2}\right)=$ $\left(w_{i}^{1}, w_{i}^{2}\right), \quad(1 \leq i \leq n)$. Again, $\left|Q_{1}(\zeta)\right| \leq n \cdot \max _{1 \leq j \leq n}\left|w_{j}^{2}-z_{j}^{2}\right| \cdot L, \quad(|\zeta|<2 R)$, which implies that

$$
|T(\xi, \zeta)-(\xi, \zeta)|=\left|\left(0, Q_{1}(\xi)\right)\right| \leq n \cdot \max _{1 \leq j \leq n}\left|w_{j}-z_{j}\right| \cdot L, \quad(|\xi|<2 R) .
$$

In particular, if $\delta=\eta$ is small enough then (4.3) holds. The equality (4.4) is clear. This completes the proof.

Remark. Lemma 3.1 holds for $\mathbb{C}^{N}, N \geq 2$. The proof is an easy elaboration of the proof above.

\section{The induction step, Part 2}

We need the following:

Lemma 5.1 Let $r>0$ and let $\Phi: \mathbb{C} \rightarrow \mathbb{C}^{2}$ be a proper holomorphic embedding. Let $\Sigma \subset \subset \mathbb{C}$ be a domain bounded by a smooth Jordan curve and assume that $\Phi(b \Sigma) \subset \mathbb{C}^{2} \backslash r \overline{\mathbb{B}}$. Then the set $(r \overline{\mathbb{B}}) \cup \Phi(\bar{\Sigma})$ is polynomially convex. 
Proof. Since $\Sigma$ is a Jordan domain with smooth boundary it is easy to see that if $K \subset \mathbb{C} \backslash \bar{\Sigma}$ is a compact set, if $a, b \in(\mathbb{C} \backslash \bar{\Sigma}) \backslash K$, and if $p$ is a path in $\mathbb{C} \backslash K$ joining $a$ and $b$ then there is a path $\tilde{p}$ in $(\mathbb{C} \backslash \bar{\Sigma}) \backslash K$ joining $a$ and $b$. Let $K=\{\zeta \in \mathbb{C} \backslash \bar{\Sigma}:|\Phi(\zeta)| \leq r\}$. Since $\Phi(b \Sigma) \subset \mathbb{C}^{2} \backslash r \overline{\mathbb{B}}$ and since $|\Phi(\zeta)| \rightarrow+\infty$ as $|\zeta| \rightarrow+\infty$, the set $K$ is compact. Suppose for a moment that $(\mathbb{C} \backslash \bar{\Sigma}) \backslash K$ is not connected. The preceding discussion implies that $\{\zeta \in \mathbb{C}:|\Phi(\zeta)|>r\}$ has a bounded component which contradicts the maximum principle. Thus, $(\mathbb{C} \backslash \bar{\Sigma}) \backslash K$ is connected which implies that for each $q \in \Phi(\mathbb{C}) \backslash(\Phi(\bar{\Sigma}) \cup r \bar{B})$ there is a path $\eta: \quad[0,1) \rightarrow \Phi(\mathbb{C}) \backslash(\Phi(\bar{\Sigma}) \cup r \overline{\mathbb{B}})$ such that $\eta(0)=q$ and $|\eta(t)| \rightarrow+\infty$ as $t \rightarrow 1$. The statement of the lemma now follows from [BF, Lemma 3.1]. This completes the proof.

Remark. It is easy to see that the proof of Lemma 3.1 in $[\mathrm{BF}]$ works for $\mathbb{C}^{N}, \quad N \geq 2$, and so Lemma 5.1. holds for proper holomorphic embeddings $\Phi: \mathbb{C} \rightarrow \mathbb{C}^{N}, N \geq 2$.

Proof of the induction step, continued. We have already mentioned that for each $m, f_{m+1}=\left(\Psi_{m+1} \circ \Theta_{m+1} \circ A_{m}\right) \circ\left(G_{m+1} \circ g_{m} \circ p_{m+1}\right)=A_{m+1} \circ g_{m+1}$. Thus, $f_{n}=H_{n} \circ g_{1} \circ\left(p_{2} \circ \cdots \circ p_{n}\right)$ where $H_{n}=\left(\Psi_{n} \circ \Theta_{n}\right) \circ \cdots \circ\left(\Psi_{2} \circ \Theta_{2}\right) \circ\left(G_{n} \circ \cdots \circ G_{2}\right)$ is a holomorphic automorphism of $\mathbb{C}^{2}$. It follows that $f_{n}\left(K_{n}\right)$ is a compact subset of $\left(H_{n} \circ g_{1}\right)(\mathbb{C})$, a closed submanifold of $\mathbb{C}^{2}$ biholomorphically equivalent to $\mathbb{C}$, whose boundary $f_{n}\left(b K_{n}\right)$ is a smooth Jordan curve which is, by (ii), contained in $\mathbb{C}^{2} \backslash r_{n} \overline{\mathbb{B}}$. By Lemma 5.1 the set $f_{n}\left(K_{n}\right) \cup r_{n} \overline{\mathbb{B}}$ is polynomially convex. By (ii) $f_{n}\left(K_{n}\right) \cup r_{n} \overline{\mathbb{B}}$ contains no point of $f_{n}\left(\left(K_{n+1} \backslash K_{n}\right) \cap S\right)$. Since $f_{n}$ is one to one it follows that $f_{n}(\xi) \neq f_{n}(\eta)$ if $\xi, \eta \in\left(K_{n+1} \backslash K_{n}\right) \cap S$. By (i), $\varphi\left(\left(K_{n+1} \backslash K_{n}\right) \cap S\right)$ does not meet $r_{n} \overline{\mathbb{B}}$. However, some points of $\varphi\left(\left(K_{n+1} \backslash K_{n}\right) \cap S\right)$ may lie in $f_{n}\left(K_{n}\right)$. Since $f_{n}\left(K_{n}\right)$ is contained in $\left(H_{n} \circ g_{1}\right)(\mathbb{C})$, a closed one dimensional complex submanifold of $\mathbb{C}^{2}$, one can change $\varphi$ slightly on $K_{n+1} \cap S$ to $\tilde{\varphi}$ so that

$$
|\tilde{\varphi}-\varphi|<\theta_{n} \text { on } K_{n+1} \cap S,
$$

so that $\tilde{\varphi}$ is one to one on $K_{n+1} \cap S$ and that $f_{n}\left(K_{n}\right) \cup r_{n} \overline{\mathbb{B}}$ contains no point of $\tilde{\varphi}\left(\left(K_{n+1} \backslash K_{n}\right) \cap S\right)$. By [FGS] there is an automorphism $\Theta_{n+1}$ of $\mathbb{C}^{2}$ which fixes each point of $f_{n}\left(K_{n} \cap S\right)$, that moves each point $f_{n}(\zeta), \zeta \in\left(K_{n+1} \backslash K_{n}\right) \cap S$ to $\tilde{\varphi}(\zeta)$, and that satisfies

$$
\left|\Theta_{n+1}-\mathrm{Id}\right|<\theta_{n} \text { on } f_{n}\left(K_{n}\right) \cup r_{n} \overline{\mathbb{B}} .
$$

By (iv) we have $f_{n}\left|K_{n} \cap S=\varphi\right| K_{n} \cap S$. Almost the same equality holds for $\Theta_{n+1} \circ f_{n}$ in place of $f_{n}$ since $\Theta_{n+1} \circ f_{n}\left|K_{n+1} \cap S=\tilde{\varphi}\right| K_{n+1} \cap S$. Applying on both sides on the left an automorphism $\Psi$ provided by Lemma 3.1 which satisfies $\Psi \circ \tilde{\varphi}=\varphi$ on $K_{n+1} \cap S$, would produce a map from $\Delta$ to $\mathbb{C}^{2}$ that would satisfy (iv) with $n$ replaced by $n+1$. However, such a map does not necessarily satisfy (ii) with $n+1$ in place of $n$ or (iii) since we have no control over what $\Theta_{n+1}$ does with $f_{n}\left(\Delta \backslash K_{n}\right)$. 


\section{The induction step, Part 3}

We perform our induction process in such a way that

$$
A_{n}\left(\left\{(z, w):|w|>T_{n} / 2\right\}\right) \text { misses } 2 r_{n-1} \mathbb{B} \text { if } n \text { is odd. }
$$

and

$$
A_{n}\left(\left\{(z, w):|z|>S_{n} / 2\right\}\right) \text { misses } 2 r_{n-1} \mathbb{B} \text { if } n \text { is even. }
$$

Recall that $\left(6.1^{\prime}\right)$ holds for $n=1$. We are describing the induction step for odd $n$ so assume that $\left(6.1^{\prime}\right)$ holds. To handle the problem described at the end of the previous section we replace $g_{n}$ in $\Theta_{n+1} \circ A_{n} \circ g_{n}=\Theta_{n+1} \circ f_{n}$ by $G_{n+1} \circ g_{n}$ where $G_{n+1}$ is an automorphism of $\mathbb{C}^{2}$ of the form $\left(2.1^{\prime}\right)$. Passing to a slightly smaller $U_{n}$ if necessary we may assume that $g_{n}\left(U_{n}\right)$ is bounded. We want that $G_{n+1}$ changes $g_{n}$ only slightly on $K_{n}$ and on $K_{n+1} \cap S$ while it maps $g_{n}\left(U_{n} \backslash \operatorname{Int} Q_{n+1}\right)$ so far from the origin that

$$
\left(\Theta_{n+1} \circ A_{n+1}\right) \circ\left(G_{n+1} \circ g_{n}\right)\left(U_{n} \backslash \operatorname{Int} Q_{n+1}\right) \subset \mathbb{C}^{2} \backslash 2 r_{n+1} \overline{\mathbb{B}}
$$

which, since $g_{n}\left(U_{n}\right)$ is bounded, and since $\Theta_{n+1} \circ A_{n+1}$ is an automorphism of $\mathbb{C}^{2}$, holds if

$$
\left|S_{n+1}\left(\frac{w}{T_{n}}\right)^{M_{n+1}}\right| \geq \rho_{n} \quad\left(|w| \geq T_{n 2}\right)
$$

provided that $\rho_{n}$ is sufficiently large. Choose $\tau_{n}>0$ so small that

$$
\left|\left(\Theta_{n+1} \circ A_{n}\right)(p)-\left(\Theta_{n+1} \circ A_{n}\right)(q)\right|<\theta_{n} \quad\left(q \in g_{n}\left(P_{n+1}\right),|p-q|<2 \tau_{n}\right) .
$$

We want that

$$
\left|S_{n+1}\left(\frac{w}{T_{n}}\right)^{M_{n+1}}\right| \leq \tau_{n} \quad\left(|w| \leq T_{n 1}\right)
$$

which will imply that $G_{n+1}$ changes $g_{n}$ on $P_{n+1}$ for at most $\tau_{n}$. Let

$$
S_{n+1}=\rho_{n}\left(\frac{T_{n}}{T_{n 2}}\right)^{M_{n+1}} .
$$

Notice that $S_{n+1}$ is arbitrarily large provided that $M_{n+1}$ is large enough. The choice of $S_{n+1}$ implies (6.3) while (6.5) becomes equivalent to

$$
\rho_{n}\left(\frac{T_{n 1}}{T_{n 2}}\right)^{M_{n+1}}<\tau_{n}
$$

which will hold provided that $M_{n+1}$ is large enough. Choose $M_{n+1}$ so large that $S_{n+1}$ becomes so large that

$$
\left(\Theta_{n+1} \circ A_{n}\right)\left(\left\{|z|>S_{n+1} / 2\right\}\right) \text { misses }\left(2 r_{n}+\varepsilon_{n}\right) \bar{B} .
$$

Notice that if an automorphism $G: \mathbb{C}^{2} \rightarrow \mathbb{C}^{2}$ satisfies $|G(z)-z|<\tau(z \in$ $R \mathbb{B})$ where $0<\tau<R$ then $(R-\tau) \overline{\mathbb{B}} \subset G(R \overline{\mathbb{B}})$. Choose a compact set 
$K_{n}^{\prime} \subset \operatorname{Int} K_{n}$ such that $f_{n}\left(\Delta \backslash K_{n}^{\prime}\right) \subset f_{n}\left(\Delta \backslash K_{n}\right)+\theta_{n} \mathbb{B}$. Now, (ii) implies that $A_{n}\left(g_{n}\left(\Delta \backslash K_{n}^{\prime}\right)\right)=f_{n}\left(\Delta \backslash K_{n}^{\prime}\right)$ misses $\left(r_{n}-\theta_{n}\right) \overline{\mathbb{B}}$ and (5.2) implies that

$$
\left(\Theta_{n+1} \circ A_{n} \circ g_{n}\right)\left(\Delta \backslash K_{n}^{\prime}\right) \subset \mathbb{C}^{2} \backslash\left(r_{n}-2 \theta_{n}\right) \overline{\mathbb{B}} .
$$

By (6.5), $\left|G_{n+1} \circ g_{n}-g_{n}\right| \leq \tau_{n}$ on $P_{n+1}$ so by (6.4)

$$
\left|\left(\Theta_{n+1} \circ A_{n} \circ G_{n+1} \circ g_{n}\right)(\zeta)-\left(\Theta_{n+1} \circ A_{n} \circ g_{n}\right)(\zeta)\right| \leq \theta_{n} \quad\left(\zeta \in P_{n+1}\right)
$$

which, by (6.9) gives

$$
\left(\Theta_{n+1} \circ A_{n} \circ G_{n+1} \circ g_{n}\right)\left(P_{n+1} \backslash K_{n}^{\prime}\right) \subset \mathbb{C}^{2} \backslash\left(r_{n}-3 \theta_{n}\right) \overline{\mathbb{B}} .
$$

Let $\zeta \in Q_{n+1} \backslash P_{n+1}$. Since $g_{n}\left(Q_{n+1} \backslash P_{n+1}\right) \subset\left\{|w|>T_{n} / 2\right\}$ and since $G_{n+1}$ does not change the $w$ coordinate we have $\left(G_{n+1} \circ g_{n}\right)(\zeta) \in\left\{|w|>T_{n} / 2\right\}$ and so $\left(A_{n} \circ G_{n+1} \circ g_{n}\right)(\zeta) \in A_{n}\left(\left\{|w|>T_{n} / 2\right\}\right)$. By $\left(6.1^{\prime}\right) A_{n}\left(\left\{|w|>T_{n} / 2\right\}\right)$ misses $2 r_{n-1} \mathbb{B}$ which implies that $\left(A_{n} \circ G_{n+1} \circ g_{n}\right)(\zeta) \in \mathbb{C}^{2} \backslash 2 r_{n-1} \mathbb{B}$. By (5.2) it follows that $\left(\Theta_{n+1} \circ A_{n} \circ G_{n+1} \circ g_{n}\right)(\zeta) \in \mathbb{C}^{2} \backslash s \overline{\mathbb{B}}$ where $s=\min \left\{r_{n}-\theta_{n}, 2 r_{n-1}-\theta_{n}\right\}$, by (3.4), satisfies $s>r_{n-1}+\theta_{n}+\varepsilon_{n}$. By (6.10), (6.2) and (3.4) it follows that

$$
\left(\Theta_{n+1} \circ A_{n} \circ G_{n+1} \circ g_{n}\right)\left(U_{n} \backslash K_{n}^{\prime}\right) \subset \mathbb{C}^{2} \backslash\left(r_{n-1}+\theta_{n}+\varepsilon_{n}\right) \overline{\mathbb{B}} .
$$

\section{The induction step, Part 4}

Note first that $\Theta_{n+1} \circ A_{n} \circ g_{n}\left|K_{n+1} \cap S=\tilde{\varphi}\right| K_{n+1} \cap S$. This does not necessarily hold if we replace $g_{n}$ by $G_{n+1} \circ g_{n}$. However, since all points of $K_{n+1} \cap S$ lie in $P_{n+1}$, since $\left|G_{n+1} \circ g_{n}-g_{n}\right|<\tau_{n}$ on $P_{n+1}$ and since $|\varphi-\tilde{\varphi}|<\theta_{n}$ on $K_{n+1} \cap S$ it follows by (6.4) that

$$
\left|\Theta_{n+1} \circ A_{n} \circ G_{n+1} \circ g_{n}-\varphi\right|<2 \theta_{n} \text { on } K_{n+1} \cap S \text {. }
$$

The problem now is that $\left(G_{n+1} \circ g_{n}\right)^{-1}\left(\left\{(z, w):|z|=S_{n+1}\right\}\right)$ is not necessarily equal to $b \Delta$ so we cannot use $\Theta_{n+1} \circ A_{n} \circ G_{n+1} \circ g_{n}$ as $f_{n+1}$ even after composing with a correction automorphism provided by Lemma 3.1. However, $\left(G_{n+1} \circ g_{n}\right)^{-1}\left(\left\{|z|=S_{n+1}\right\}\right)$ is a real analytic curve that is arbitrarily small $\mathcal{C}^{1}$ perturbation of $b \Delta$ independently of $M_{n+1}$ if only $S_{n+1}$ is large enough [G, Sec. 5]; in our case this means if only $M_{n+1}$ is large enough.

Thus, provided that $M_{n+1}$ is large enough the conformal map $p_{n+1}$ mapping $\Delta$ to the domain $\left(G_{n+1} \circ g_{n}\right)^{-1}\left(\left\{|z|<S_{n+1}\right\}\right)$ and satisfying $p_{n+1}(0)=$ $0, p_{n+1}^{\prime}(0)>0$, is arbitrarily close to the identity on $\Delta$ provided that $M_{n+1}$ is sufficiently large [P, p. 286]. Once we have chosen $M_{n+1}$ the map $p_{n+1}$ extends holomorphically to a neighbourhood $U_{n+1} \subset U_{n}$ of $\bar{\Delta}$ so that the extended map $p_{n+1}$ maps $U_{n+1}$ biholomorphically onto $p_{n+1}\left(U_{n+1}\right)$ and so that the map $g_{n+1}=G_{n+1} \circ g_{n} \circ p_{n+1}: U_{n+1} \rightarrow \mathbb{C}^{2}$ is transverse to $\left\{(z, w):|z|=S_{n+1}\right\}$ and satisfies $g_{n+1}^{-1}\left(\left\{|z|=S_{n+1}\right\}\right)=b \Delta[\mathrm{G}]$.

Passing to a larger $M_{n+1}$ if necessary we may assume that $p_{n+1}$ is so close to the identity on $\bar{\Delta}$ that

$$
\left|g_{n} \circ p_{n+1}-g_{n}\right|<\tau_{n} \text { on } \bar{\Delta}
$$


and that

$$
\begin{cases}K_{n} \subset p_{n+1}^{-1}\left(P_{n+1}\right), & K_{n+1} \cap S \subset p_{n+1}^{-1}\left(P_{n+1}\right) \\ p_{n+1}^{-1}\left(Q_{n+1}\right) \subset \operatorname{Int} K_{n+1}, & p_{n+1}^{-1}\left(K_{n}^{\prime}\right) \subset K_{n} .\end{cases}
$$

Since $\left|G_{n+1} \circ g_{n}-g_{n}\right| \leq \tau_{n}$ on $P_{n+1}$ it follows that $\left|G_{n+1} \circ g_{n} \circ p_{n+1}-g_{n} \circ p_{n+1}\right| \leq$ $\tau_{n}$ on $p_{n+1}^{-1}\left(P_{n+1}\right)$ which, by $(7.2)$ and (7.3) implies that

$$
\left|G_{n+1} \circ g_{n} \circ p_{n+1}-g_{n}\right|<2 \tau_{n} \text { on } K_{n} \cup\left(K_{n+1} \cap S\right) \text {. }
$$

Since $K_{n} \cup\left(K_{n+1} \cap S\right) \subset P_{n+1},(6.4)$ implies that

$\left|\left(\Theta_{n+1} \circ A_{n} \circ G_{n+1} \circ g_{n} \circ p_{n+1}\right)(\zeta)-\left(\Theta_{n+1} \circ A_{n} \circ g_{n}\right)(\zeta)\right|<\theta_{n}\left(\zeta \in K_{n} \cup\left(K_{n+1} \cap S\right)\right)$.

By (5.2), $\left|\Theta_{n+1} f_{n}(\zeta)-f_{n}(\zeta)\right|<\theta_{n}\left(\zeta \in K_{n}\right)$ so it follows that

$$
\left|\left(\Theta_{n+1} \circ A_{n} \circ G_{n+1} \circ g_{n} \circ p_{n+1}\right)(\zeta)-f_{n}(\zeta)\right|<2 \theta_{n} \quad\left(\zeta \in K_{n}\right) \text {. }
$$

Further, since $\left(\Theta_{n+1} \circ A_{n} \circ g_{n}\right) \mid K_{n+1} \cap S=\tilde{\varphi}$ and since $|\tilde{\varphi}-\varphi|<\theta_{n}$ on $K_{n+1} \cap S$ it follows also that

$$
\left|\left(\Theta_{n+1} \circ A_{n} \circ G_{n+1} \circ g_{n} \circ p_{n+1}\right)(\zeta)-\varphi(\zeta)\right|<3 \theta_{n} \quad\left(\zeta \in K_{n+1} \cap S\right) .
$$

The choice of $R$ and (3.3) imply that there is a holomorphic automorphism $\Psi_{n+1}$ of $\mathbb{C}^{2}$ such that

$$
\left|\Psi_{n+1}-\mathrm{Id}\right|<\varepsilon_{n} / 2^{n+1} \text { on } R \mathbb{B}
$$

and such that

$$
\left(\Psi_{n+1} \circ \Theta_{n+1} \circ A_{n} \circ G_{n+1} \circ g_{n} \circ p_{n+1}\right)(\zeta)=\varphi(\zeta) \quad\left(\zeta \in K_{n+1} \cap S\right) .
$$

Put $f_{n+1}=A_{n+1} \circ g_{n+1}$, where $A_{n+1}=\Psi_{n+1} \circ \Theta_{n+1} \circ A_{n}$, and $g_{n+1}=G_{n+1} \circ$ $g_{n} \circ p_{n+1}$. By (7.7), (iv) is satisfied with $n+1$ in place of $n$. Since $\theta_{n}<\varepsilon_{n} / 2^{n+2}$ and since $f_{n}\left(K_{n}\right)+\mathbb{B} \subset R \mathbb{B},(7.4)$ and (7.6) imply that $\left|f_{n+1}(\zeta)-f_{n}(\zeta)\right|<$ $2 \theta_{n}+\varepsilon_{n} / 2^{n+1}<\varepsilon_{n} / 2^{n}\left(\zeta \in K_{n}\right)$ so that (v) is satisfied.

By (7.3), $\zeta \in \Delta \backslash \operatorname{Int} K_{n+1}$ implies that $p_{n+1}(\zeta) \in U_{n} \backslash Q_{n+1}$ which, by (6.2) implies that $\left(\Theta_{n+1} \circ A_{n} \circ g_{n+1}\right)(\zeta) \in \mathbb{C}^{2} \backslash 2 r_{n+1} \overline{\mathbb{B}}$. By (7.6), by the fact that $R>2 r_{n+1}$ and by $(3.2)$ it follows that $f_{n+1}(\zeta) \in \mathbb{C}^{2} \backslash\left(2 r_{n+1}-\varepsilon_{n} / 2^{n+1}\right) \mathbb{B} \subset$ $\mathbb{C}^{2} \backslash\left(2 r_{n+1}-r_{1}\right) \mathbb{B} \subset \mathbb{C}^{2} \backslash\left(r_{n+1} \overline{\mathbb{B}}\right)$. Thus (ii) holds with $n$ replaced by $n+1$.

By $(6.11)$

$$
\left(\Theta_{n+1} \circ A_{n} \circ G_{n+1} \circ g_{n}\right)\left(U_{n} \backslash K_{n}^{\prime}\right) \subset \mathbb{C}^{2} \backslash\left(r_{n-1}+\theta_{n}+\varepsilon_{n}\right) \overline{\mathbb{B}} .
$$

If $\zeta \in \Delta \backslash K_{n}$ then, by (7.3), $p_{n+1}(\zeta) \in p_{n+1}(\Delta) \backslash K_{n}^{\prime} \subset U_{n} \backslash K_{n}^{\prime}$ so

$$
\left(\Theta_{n+1} \circ A_{n} \circ g_{n+1}\right)\left(\Delta \backslash K_{n}\right) \subset \mathbb{C}^{2} \backslash\left(r_{n-1}+\theta_{n}+\varepsilon_{n}\right) \overline{\mathbb{B}},
$$

and since $R_{n-1}+\theta_{n}+\varepsilon_{n}<R$ it follows by (7.6) that $f_{n+1}\left(\Delta \backslash K_{n}\right) \subset \mathbb{C}^{2} \backslash r_{n-1} \overline{\mathbb{B}}$, that is, (iii) is satisfied.

Finally, (6.8) implies that

$$
\left(\Psi_{n+1} \circ \Theta_{n+1} \circ A_{n}\right)\left(\left\{|z|>S_{n+1} / 2\right\}\right) \text { misses } \Psi_{n+1}\left(\left(2 r_{n}+\varepsilon_{n}\right) \overline{\mathbb{B}}\right) .
$$

Since $2 r_{n}+\varepsilon_{n}<R,(7.6)$ implies that $2 r_{n} \mathbb{B} \subset \Psi_{n+1}\left(\left(2 r_{n}+\varepsilon_{n}\right) \mathbb{B}\right)$ so $A_{n+1}(\{|z|>$ $\left.\left.S_{n+1} / 2\right\}\right)$ misses $2 r_{n} \mathbb{B}$, that is, $\left(6.1^{\prime \prime}\right)$ holds with $n$ replaced by $n+1$. 
This completes the proof of the induction step.

Since the map $\varphi$ is proper, (vii) and the fact that (3.1) holds for every $n$ imply that $r_{n} \rightarrow+\infty$ as $n \rightarrow \infty$. The proof of Theorem 1.1 is complete.

\section{Remarks}

Theorem 1.1 holds with $\mathbb{C}^{2}$ replaced by $\mathbb{C}^{N}, N \geq 2$.

Theorem 8.1 Let $N \geq 2$. Given a discrete set $S \subset \Delta$ and a proper injection $\varphi: S \rightarrow \mathbb{C}^{N}$ there is a proper holomorphic embedding $f: \Delta \rightarrow \mathbb{C}^{N}$ that extends $\varphi$.

If $N \geq 3$ then one proves Theorem 8.1. as in the case $N=2$ with a slight modification: Let $\iota: \mathbb{C}^{2} \rightarrow \mathbb{C}^{N}$ be the standard embedding $\iota\left(\zeta_{1}, \zeta_{2}\right)=$ $\left(\zeta_{1}, \zeta_{2}, 0, \cdots, 0\right)$. In the proof we replace $f_{n}=A_{n} \circ g_{n}$ by $f_{n}=A_{n} \circ \iota \circ g_{n}$ where $A_{n}$ is a holomorphic automorphism of $\mathbb{C}^{N}$ and $g_{n}$, as in the proof in the case $N=2$, is a one to one and regular holomorphic map from an open neighbourhood $U_{n}$ of $\bar{\Delta}$ to $\mathbb{C}^{2}$ which, for even $n$ is transverse to $\left\{(z, w):|z|=S_{n}\right\}$ and satisfies $g_{n}^{-1}\left(\left\{|z|=S_{n}\right\}\right)=b \Delta$, and for odd $n$, is transverse to $\left\{(z, w):|w|=T_{n}\right\}$ and satisfies $g_{n}^{-1}\left(\left\{|w|=T_{n}\right\}\right)=b \Delta$. Also, in the induction step, the maps $\Theta_{n+1}$ and $\Psi_{n+1}$ are automorphisms of $\mathbb{C}^{N}$ and $G_{n+1}$ is an automorphism of $\mathbb{C}^{2}$. In $\left(6.1^{\prime}\right)$ and $\left(6.1^{\prime \prime}\right)$ we replace $A_{n}$ by $A_{n} \circ \iota$.

We say that two proper holomorphic embeddedings $f_{1}, f_{2}: \Delta \rightarrow \mathbb{C}^{N}$ are $\operatorname{Aut}\left(\mathbb{C}^{N}\right)$-equivalent if there is an automorphism $\Psi: \mathbb{C}^{N} \rightarrow \mathbb{C}^{N}$ such that $f_{2}=\Psi \circ f_{1}$.

Corollary 8.2 Let $N \geq 2$. The set of Aut $\left(\mathbb{C}^{N}\right)$-equivalence classes of proper holomorphic embeddedings of $\Delta$ into $\mathbb{C}^{N}$ is uncountable.

Proof. [BFo] It is known [RR, Remark 5.2] that there is an uncountable family $E$ of discrete injective sequences in $\mathbb{C}^{N}$ such that if $\left\{z_{n}, n \in \mathbb{N}\right\},\left\{w_{n}, n \in \mathbb{N}\right\}$ are distinct elements of $E$ then there is no automorphism $\Psi$ of $\mathbb{C}^{N}$ such that $\Psi\left(z_{n}\right)=w_{n} \quad(n \in \mathbb{N})$. Let $\left\{\zeta_{n}\right\} \subset \Delta$ be an injective sequence, $\lim _{n \rightarrow \infty}\left|\zeta_{n}\right|=1$, and let $\left\{z_{n}, n \in \mathbb{N}\right\},\left\{w_{n}, n \in \mathbb{N}\right\}$ be distinct elements of $E$. By Theorem 8.1 there are proper holomorphic embeddings $f_{1}, f_{2}: \Delta \rightarrow \mathbb{C}^{N}$ such that $f_{1}\left(\zeta_{j}\right)=$ $z_{j}, f_{2}\left(\zeta_{j}\right)=w_{j}(j \in \mathbb{N})$. Every automorphism $\Psi$ of $\mathbb{C}^{N}$ such that $f_{2}=\Psi \circ f_{1}$ would have to satisfy $\Psi\left(z_{n}\right)=w_{n} \quad(n \in \mathbb{N})$ and there is no such $\Psi$. Thus, in this way, each element of $E$ produces a proper holomorphic embedding of $\Delta$ into $\mathbb{C}^{N}$ and the embeddings associated with distinct elements of $E$ are not $\operatorname{Aut}\left(\mathbb{C}^{N}\right)$-equivalent. This completes the proof.

\section{Acknowledgements}

The author is indebted to Frank Kutzschebauch for a helpful remark.

This work was supported in part by a grant from the Ministry of Science and Technology of the Republic of Slovenia. A part of work was done in January 
2000 during the author's visit at Bar Ilan University, Ramat Gan, Israel which was supported by a Slovene-Israeli grant.

\section{References}

[A] H. Alexander, Explicit imbedding of the (punctured) disc into $\mathbb{C}^{2}$, Comment. Math. Helv. 52 (1977), 539-544.

[BF] G. T. Buzzard, J. E. Fornaess, An embedding of $\mathbb{C}$ into $\mathbb{C}^{2}$ with hyperbolic complement, Math. Ann. 306 (1996), 539-546.

[BFo] G. T. Buzzard, F. Forstnerič, A Carleman type theorem for proper holomorphic embeddings, Ark. Mat. 35 (1997), 157-169.

[DK] H. Derksen, F. Kutzschebauch, Global holomorphic linearization of actions of compact Lie groups on $\mathbb{C}^{n}$, Contemp. Math. 222 (1999), 201-210.

[FGR] F. Forstnerič, J. Globevnik, J.-P. Rosay, Nonstraightenable complex lines in $\mathbb{C}^{2}$, Ark. Mat. 34 (1996), 97-101.

[FGS] F. Forstnerič, J. Globevnik, B. Stensones, Embedding holomorphic discs through discrete sets, Math. Ann. 305 (1996), 559-569.

[G] J. Globevnik, On Fatou-Bieberbach domains, Math. Z. 229 (1998), 91-106.

[GS] J. Globevnik, B. Stensones, Holomorphic embeddings of planar domains into $\mathbb{C}^{2}$, Math. Ann. 303 (1995), 579-597.

[Na] R. Narasimhan, Imbedding of holomorphically complete complex spaces, Amer. J. Math. 82 (1960), 917-934.

[P] Ch. Pommerenke, Univalent functions, Vandenhoeck \& Ruprecht, Göttingen, 1975.

$[\mathrm{RR}]$ J.-P. Rosay, W. Rudin, Holomorphic maps from $\mathbb{C}^{n}$ to $\mathbb{C}^{n}$, Trans. Amer. Math. Soc. $\mathbf{3 1 0}$ (1988), 47-86.

[St] J.-L. Stehle, Plongements du disque dans $\mathbb{C}^{2}$, Séminaire Pierre Lelong (Analyse), Année 1970-1971, pp. 119-130. Lecture Notes in Math., Vol. 275, Springer, Berlin, 1972.

Institute of Mathematics, Physics and Mechanics, University of Ljubluana, LjublJana, Slovenia.

E-mail address: josip.globevnik@fmf.uni-lj.si 